\title{
Modern Management of Hypertensive Emergencies
}

\author{
Monica Marton-Popovici \\ Department of Anesthesiology and Critical Care, Swedish Medical Center, Seattle, USA
}

\begin{abstract}
Hypertensive emergencies (HE) represent critical conditions in which extremely high blood pressure values are accompanied by acute hypertension-mediated organ damage. In this clinical setting, the main therapeutic goal is represented by the immediate reduction of blood pressure, in order to limit the extension or promote the regression of target organ damage. At present, HE are classified according to the condition or target organ involved, into: (1) malignant hypertension with or without thrombotic microangiopathy; (2) coronary ischemia or acute cardiogenic pulmonary edema; (3) acute stroke or hypertensive encephalopathy; (4) acute aortic dissection or aneurysm; and (5) eclampsia or severe preeclampsia/HELLP syndrome. The management of these conditions is different in relation to the complex pathophysiology involved in each of these types. This mini-review presents the main characteristics and management strategy for different forms of $\mathrm{HE}$, revealing the particularities of management strategy for each of them.
\end{abstract}

Keywords: hypertension, blood pressure, target organ damage

\section{ARTICLE HISTORY}

Received: November 17, 2019 Accepted: December 16, 2019

\section{CORRESPONDENCE}

Monica Marton-Popovici

Swedish Medical Center, Department of Internal Medicine and Critical Care 21601, 76th Ave W, Edmonds, Washington, 98026, USA. Tel: +1 (202) 4256404000 E-mail: monica.marton-popovici@ swedish.org
Hypertensive emergencies (HE) are critical conditions associated with significant disability and high healthcare costs. Even though the mortality rates associated with these emergencies have decreased over the last years, patients with HE are still at a high risk. ${ }^{1-3}$ Kumar et al. found that $16 \%$ of patients admitted to hospital for a HE had an unplanned readmission within 30 days. ${ }^{4}$

According to the latest definition of the European Society of Cardiology (ESC), HE represent critical conditions in which extremely high blood pressure (BP) values are accompanied by acute hypertension-mediated organ damage including the heart, brain, kidney, retina, or large

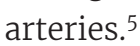

The incidence of $\mathrm{HE}$ is constantly increasing. Janke et al. reported that the number of visits to the emergency department for HE has more than doubled from 2006 to $2013 .{ }^{6}$ In a large study on 129,914 admissions, Shah et al. reported a rising trend in HE hospitalization over 10 years, increasing from 9,511 to 15,479 ( $\mathrm{p}<0.001$ ), in parallel with a significant reduction in long-term mortality (from $0.8 \%$ to $0.3 \%, p<0.001) .{ }^{7}$ However, the mortality of patients with HE remains high. A study by Guiga et al. reported an in-hospital mortality rate of $12.5 \%$ for HE compared to $1.8 \%$ for hypertensive urgencies. ${ }^{8}$ As this condition is life-threatening, the main therapeutic goal in this clinical setting is represented by the immediate reduction of blood pressure, in order to limit the extension or promote the regression of target organ damage..$^{9,10}$

It is important to differentiate this condition from the classic uncontrolled hypertension without target organ damage, as the management of this entity is completely different. According to ESC guidelines, the involvement of target organs is mandatory to include the patient in the group of HE, while patients with severe hypertension in whom there is no sign of target organ damage are classified as "hypertensive urgencies". 5

In a study on 2,011 patients presenting to the emergency department, Maghraoui et al. found that $22.8 \%$ of patients presented high BP at arrival, decreasing to $18.5 \%$ after 10 minutes at rest. ${ }^{11}$ Salvetti et al., in a study on a large database of patients presenting with high BP in the emergency department over a period of 7 years, showed that HE was 
present in $20.4 \%$ of cases in 2008 and in $15.4 \%$ of cases in 2015 , while the rate of hypertensive urgencies increased from $79.6 \%$ in 2008 to $84.5 \%$ in $2015 .{ }^{12}$ Salagre et al. reported significantly higher rates of HE, being encountered in $48 \%$ of cases with elevated BP presenting to the emergency department, while $51 \%$ of cases were hypertensive urgencies. ${ }^{13}$ In another study, Pierin et al. evaluated a total of 508 patients presenting at the emergency department with a hypertensive crisis and reported that $71.7 \%$ of patients presented hypertensive urgency, 19.1\% HE, and $9.2 \%$ hypertensive pseudocrisis. Interestingly, they reported that while HE were associated with neurological problems and advanced age, hypertensive urgencies were associated with headache, and hypertensive pseudocrisis was linked to pain or emotional issues. ${ }^{14}$ Nakalema et al. found also that HE are associated with poor compliance to prescribed medication. ${ }^{15}$

The mechanisms involved in the progression from hypertension to target organ damage are complex. A severe uncontrolled hypertension leads to increase in renal vascular resistance and to pressure-induced natriuresis, which further increases vascular resistance. The sudden increase in vascular resistance and pressure-induced natriuresis triggers a vicious circle, which ultimately results in microcirculatory damage with consequent target organ damage. Another finding was reported by Sansone et al., who described an increased release of circulating endothelial microparticles in patients with HE, suggesting a link between physico-mechanical injury and activation, and decreased wall shear stress. ${ }^{16}$

Several clinical settings associated with $\mathrm{HE}$ are also included in the recent definition released by the ESC. Malignant hypertension, for instance, is defined as a HE in which a severe BP elevation is associated with advanced retinopathy, documented by retinal hemorrhage, papillary edema, or cotton wool spots. ${ }^{17-20}$ Hypertensive encephalopathy is another type of HE in which a severe hypertension is associated with seizures, lethargy, cortical blindness, or coma. A more rarely recognized clinical condition is thrombotic angiopathy, in which severe elevations of $\mathrm{BP}$ are associated with Coombs-negative hemolysis and thrombocytopenia, which regress after controlling BP values. ${ }^{5}$

According to the guidelines of the American Heart Association, the criteria for the definition of $\mathrm{EH}$ are the increase of systolic BP values above $180 \mathrm{mmHg}$ and diastolic BP values above $120 \mathrm{mmHg}$, associated with new, progressive, or worsening target organ damage..$^{21}$ In the absence of target organ damage, the condition is classified as markedly elevated BP and treated by adjustment of oral antihypertensive treatment, while in the presence of tar- get organ damage it is recommended to admit the patient to an intensive care unit. After admission, the treatment strategy is different according to the type of HE. Generally, it is recommended to reduce the BP by $25 \%$ in the first hour, then to below $160 / 100 \mathrm{mmHg}$ over the next 2-6 hours, and then to normal over the next $24-48$ hours. ${ }^{21}$ However, in the presence of aortic dissection, pheochromocytoma, or severe preeclampsia or eclampsia, it is recommended to reduce the systolic BP to values lower than $140 \mathrm{mmHg}$ in the first hour, and below $120 \mathrm{mmHg}$ in aortic dissection. ${ }^{21}$

The clinical presentation of patients with HE depends on the type of organ damage. A study by Dhadke et al. reported that the most common complaint was breathlessness in 34\% of patients, followed by neurological deficit in $28 \%$ of patients. ${ }^{22}$ In another study, Saladini et al. showed that the symptoms most suggestive of organ damage in patients with HE are chest pain (89\%), visual disturbances $(89.8 \%)$, dyspnea $(82 \%)$, and headache $(82 \%) .{ }^{23}$

The ESC classifies HE according to the condition or target organ involved into: (1) malignant hypertension with or without thrombotic microangiopathy; (2) coronary ischemia or acute cardiogenic pulmonary edema; (3) acute stroke or hypertensive encephalopathy; (4) acute aortic dissection or aneurysm; and (5) eclampsia or severe preeclampsia/HELLP syndrome. The management of these conditions is different in relation to the complex pathophysiology involved in each of these types. ${ }^{5}$

Malignant hypertension with or without thrombotic microangiopathy is characterized by a sudden increase in BP values above 180/110 mmHg, associated with cephalea and visual symptoms. The recommended tests for this condition consist in fundoscopy to detect hypertensive retinopathy and cognitive function testing in patients with symptoms of cognitive impairment. The main criteria used for defining kidney involvement as a target organ in hypertension include impaired renal function, an increase in creatinine levels of $>30 \%$, and proteinuria. ${ }^{24}$ These changes can be easily detected by performing laboratory analysis such as urine albumin/creatinine ratio to detect elevations in albumin excretion, or blood creatinine and eGFR to detect renal disease. At the same time, an abdominal ultrasound can evaluate renal structure and size, and can exclude the presence of renovascular disease. In malignant hypertension, the immediate treatment should decrease the BP by $25 \%$ within several hours, using labetalol or nicardipine as first-line treatment, or nitroprusside and urapidil as alternative therapies. ${ }^{5}$

Coronary ischemia or acute cardiogenic pulmonary edema: the association between a HE and a cardiac disease 
represents an extremely dangerous condition, as a sudden increase in BP in a coronary patient can lead to severe forms of acute coronary syndromes or acute heart failure. In a recent study, Hassan et al. found a direct link between in-hospital BP variability and major cardiovascular events in patients with acute coronary syndromes. ${ }^{25}$ The main criteria used for defining heart involvement as a target organ in hypertension are represented by marked left ventricular hypertension, impaired systolic function, increased troponin levels, and ischemic changes on the ECG. 5,24

The diagnostic algorithm in the case of a patient presenting with high BP values (above 180/110 $\mathrm{mmHg}$ ) and chest pain should focus on the identification of any signs of coronary ischemia, using basic tests such as ECG, troponin, and $\mathrm{CK}-\mathrm{MB}$, or more advanced ones such as coronary angiography or coronary CT angiography. Also, echocardiography could be used in emergency settings, to assess cardiac structure, the presence of left ventricular wall hypertrophy, and the alteration of systolic or diastolic function. Another situation is represented by the presentation of a patient with high BP values and acute dyspnea, suggesting acute heart failure associated with a HE. In this case, X-ray and lung ultrasound, together with the determination of serum levels of NT-proBNP, could serve to identify the ventricular damage caused by the elevated BP.

In case of an acute coronary event associated to high $\mathrm{BP}$, the initial management should focus on the immediate reduction of systolic BP to values below $140 \mathrm{mmHg}$, using intravenous infusion of nitroglycerine or labetalol as firstline treatment, or urapidil as an alternative. In the case of an acute cardiogenic pulmonary edema, systolic BP should be immediately reduced to values lower than $140 \mathrm{mmHg}$ using intravenous nitroprusside or nitroglycerine associated with a loop diuretic as first-line treatment.

Acute stroke or hypertensive encephalopathy is another condition classified as a HE. The main criteria used to define the brain as target organ involvement in HE are represented by clinical presentation with ischemic or hemorrhagic stroke, or extensive white matter lesions. In uncertain cases, carotid ultrasound could help to identify or exclude the presence of a carotid plaque or stenosis as a possible cause of ischemic stroke, independent of the level of BP.

According to American guidelines, whenever a patient with ischemic stroke and elevated BP qualifies for intravenous thrombolysis, the immediate treatment should lower systolic BP to $<185 \mathrm{mmHg}$ and diastolic $\mathrm{BP}$ to $<110 \mathrm{mmHg}$ before the initiation of intravenous thrombolysis, and maintain a BP $<180 / 105 \mathrm{mmHg}$ for the first 24 hours after iv thrombolysis. ${ }^{21}$ However, when the patient does not qualify for intravenous thrombolysis, the recommended treatment strategy is different according to the $\mathrm{BP}$ values: for values $>220 / 110 \mathrm{mmHg}$, it is recommended to lower the BP by $15 \%$ in the first 24 hours from treatment initiation, while for values $<220 / 110 \mathrm{mmHg}$, routine immediate BP lowering is not recommended. Instead, it is recommended to initiate or reinitiate the antihypertensive treatment only after neurological stability.

In patients with acute intracerebral hemorrhage and BP $>180 \mathrm{mmHg}$, immediate lowering of systolic BP to levels between 130 and $180 \mathrm{mmHg}$ is recommended, using labetalol and nicardipine as first-line therapy. ${ }^{5,21}$

Acute aortic dissection or aneurysm is a critical cardiac emergency, with an extremely high mortality in acute settings. In any suspicion of aortic dissection, aortic imaging using echocardiography, transesophageal echocardiography, or CT angiography should be performed in emergency conditions to establish the diagnosis, as the survival is critically dependent on the prompt initiation of adequate therapeutic measures.

In case of acute aortic dissection, immediate reduction of systolic BP to $<120 \mathrm{mmHg}$ and of heart rate to $<60 /$ minute are recommended, using esmolol and nitroprusside or nitroglycerine or nicardipine as first-line treatment., ${ }^{5,21}$

Eclampsia or severe preeclampsia/HELLP syndrome are severe forms of HE in which a pregnant woman after 20 weeks of gestation presents with proteinuria $(\geq 300 \mathrm{mg} /$ 24 -hour urine collection) or a protein/creatinine ratio $>0.3$, associated with a systolic BP $>140 \mathrm{mmHg}$ or a diastolic BP $>90 \mathrm{mmHg}$. In the absence of proteinuria, other criteria used to define eclampsia or severe preeclampsia in the presence of new-onset hypertension are: thrombocytopenia (platelet count $<100,000 / \mu \mathrm{L}$ ), renal insufficiency, or elevated liver transaminases. ${ }^{5}$ As this condition is associated with an increased maternal-fetal risk, antihypertensive treatment should be initiated as soon as possible in order to control BP values and decrease the risk of fetal death. Labetalol should be considered as a first-line agent whenever available, while hydralazine and nifedipine can be considered as second-line therapy. According to European guidelines, any systolic BP value $>170 \mathrm{mmHg}$ or diastolic BP value $>110 \mathrm{mmHg}$ in a pregnant woman represents an emergency that requires immediate hospitalization. In patients considered at moderate-to-high risk of preeclampsia, it is recommended to initiate the treatment with low-dose aspirin from week $12 .{ }^{26,27}$ At the same time, delivery is recommended at 37 weeks in women with gestational hypertension or mild preeclampsia. ${ }^{28}$ Shields et al. demonstrated that an early standardized treatment of BP elevations may lead to a significant reduction of eclampsia and maternal risk. ${ }^{29}$ 


\section{CONCLUSIONS}

HE represent a frequent type of cardiovascular emergency associated with high risk. In acute settings, the distinction between $\mathrm{HE}$ and hypertensive urgencies is essential for a proper management of the patient at risk. The involvement of target organ damage is essential for defining a $\mathrm{HE}$, an acute clinical condition which requires immediate hospital admission and initiation of therapies specific for each type of organ involved.

\section{CONFLICT OF INTEREST}

Nothing to declare.

\section{REFERENCES}

1. Lane DA, Lip GY, Beevers DG. Improving survival of malignant hypertension patients over 40 years. Am J Hypertens. 2009;22:1199-1204. doi: 10.1038/ajh.2009.153.

2. Amraoui F, Van Der Hoeven NV, Van Valkengoed IG, Vogt L, Van Den Born BJ. Mortality and cardiovascular risk in patients with a history of malignant hypertension: a case-control study. J Clin Hypertens (Greenwich). 2014;16:122-126. doi: 10.1111/jch.12243.

3. Gonzalez R, Morales E, Segura J, Ruilope LM, Praga M. Longterm renal survival in malignant hypertension. Nephrol Dial Transplant. 2010;25:3266-3272. doi: 10.1093/ndt/gfq143.

4. Kumar N, Simek S, Garg N, et al. Thirty-Day Readmissions After Hospitalization for Hypertensive Emergency. Hypertension. 2019;73:60-67. doi: 10.1161/HYPERTENSIONAHA.118.11691.

5. Williams B, Mancia G, Spiering W, et al. 2018 ESC/ESH Guidelines for the management of arterial hypertension The Task Force for the management of arterial hypertension of the European Society of Cardiology (ESC) and the European Society of Hypertension (ESH). Eur Heart J. 2018:39;30213104. doi:10.1093/eurheartj/ehy339.

6. Janke AT, McNaughton CD, Brody AM, Welch RD, Levy PD. Trends in the Incidence of Hypertensive Emergencies in US Emergency Departments From 2006 to 2013. J Am Heart Assoc. 2016;5:e004511. doi: 10.1161/JAHA.116.004511.

7. Shah M, Patil S, Patel B, et al. Trends in Hospitalization for Hypertensive Emergency, and Relationship of EndOrgan Damage With In-Hospital Mortality. Am J Hypertens. 2017;30:700-706. doi: 10.1093/ajh/hpx048.

8. Guiga H, Decroux C, Michelet P, et al. Hospital and out-ofhospital mortality in 670 hypertensive emergencies and urgencies. J Clin Hypertens (Greenwich). 2017;19:1137-1142. doi: 10.1111/jch.13083.

9. van den Born BJ, Lip GYH, Brguljan-Hitij J, et al. ESC Council on hypertension position document on the management of hypertensive emergencies. Eur Heart J Cardiovasc Pharmacotherapy. 2019;5:37-46. doi: 10.1093/ehjcvp/pvy032.

10. Vaughan CJ, Delanty N. Hypertensive emergencies. Lancet 2000;356:411-417.

11. Maghraoui H, Zaouche K, Yahya Y, Mghirbi A, Ben Hamida G, Majed K. Care of the hypertensive urgencies and emergencies in the emergency departement. Tunis Med. 2019;97:468-475.

12. Salvetti M, Paini A, Colonetti E, et al. Hypertensive emergencies and urgencies: a single-centre experience in Northern Italy 2008-2015. J Hypertens. 2020;3:52-58. doi: 10.1097/HJH.0000000000002213.

13. Salagre SB, Itolikar SM, Gedam K. A Prospective, Observational Study to Determine the Prevalence and Clinical Profile of Patients of Hypertensive Crisis in a Tertiary Care Hospital. J Assoc Physicians India. 2017;65:14-21.

14. Pierin AMG, Flórido CF, Santos JD. Hypertensive crisis: clinical characteristics of patients with hypertensive urgency, emergency and pseudocrisis at a public emergency department. Einstein (Sao Paulo). 2019;17:eAO4685. doi: 10.31744/einstein_journal/2019AO4685.

15. Nakalema I, Kaddumukasa M, Nakibuuka J, et al. Prevalence, patterns and factors associated with hypertensive crises in Mulago hospital emergency department; a cross-sectional study. Afr Health Sci. 2019;19:1757-1767. doi: 10.4314/ahs. v19i1.52.

16. Sansone R, Baaken M, Horn P, et al. Release of endothelial microparticles in patients with arterial hypertension, hypertensive emergencies and catheter-related injury. Atherosclerosis. 2018;273:67-74. doi: 10.1016/j. atherosclerosis.2018.04.012.

17. Chester EM, Agamanolis DP, Banker BQ Victor M. Hypertensive encephalopathy: a clinicopathologic study of 20 cases. Neurology. 1978;28:928-939.

18. van den Born BJ, Koopmans RP, Groeneveld JO, van Montfrans GA. Ethnic disparities in the incidence, presentation and complications of malignant hypertension. J Hypertens 2006;24:2299-2304.

19. Pinna G, Pascale C, Fornengo P, et al. Hospital admissions for hypertensive crisis in the emergency departments: a large multicenter Italian study. PLoS One. 2014;9:e93542. doi: 10.1371/journal.pone.0093542.

20. van den Born BJ, Lowenberg EC, van der Hoeven NV, et al. Endothelial dysfunction, platelet activation, thrombogenesis and fibrinolysis in patients with hypertensive crisis. J Hypertens 2011;29:922-927. doi: 10.1097/HJH.obo13e328345023d.

21. Whelton PK, Carey RM, Aronow WS, et al. Guideline for the Prevention, Detection, Evaluation, and Management of High Blood Pressure in Adults: Executive Summary A Report of the American College of Cardiology/American Heart Association Task Force on Clinical Practice Guidelines. Hypertension. 2018;71:1269-1324. doi: 10.1161/HYP.0000000000000066.

22. Dhadke SV, Dhadke VN, Batra DS. Clinical Profile of Hypertensive Emergencies in an Intensive Care Unit. J Assoc Physicians India. 2017;65:18-22.

23. Saladini F, Mancusi C, Bertacchini F, et al. Diagnosis and treatment of hypertensive emergencies and urgencies among Italian emergency and intensive care departments. Results from an Italian survey: Progetto GEAR (Gestione dell'Emergenza e urgenza in ARea critica). Eur J Intern Med. 2019;pii:So953-6205(19)30341-3. doi: 10.1016/j. ejim.2019.10.004.

24. Cremer A, Amraoui F, Lip GY, et al. From malignant hypertension to hypertension-MOD: a modern definition for an old but still dangerous emergency. J Hum Hypertens 2016;30:463-466. doi: 10.1038/jhh.2015.112.

25. Hassan AKM, Abd-El Rahman H, Mohsen K, Dimitry SR. Impact of in-hospital blood pressure variability on cardiovascular outcomes in patients with acute coronary 
syndrome. J Clin Hypertens (Greenwich). 2017;19:1252-1259. doi: 10.1111/jch.13107.

26. MacDonald TM, Williams B, Webb DJ, et al. Combination therapy is superior to sequential monotherapy for the initial treatment of hypertension: a double-blind randomized controlled trial. J Am Heart Assoc. 2017;6:e006986. doi: 10.1161/JAHA.117.006986.

27. Yusuf S, Lonn E, Pais P, Bosch J, et al. Blood-pressure and cholesterol lowering in persons without cardiovascular disease. N Engl J Med. 2016;374: 2032-2043. doi: 10.1056/ NEJMoa1600177.
28. Koopmans CM, Bijlenga D, Groen $\mathrm{H}$, et al. Induction of labour versus expectant monitoring for gestational hypertension or mild pre-eclampsia after 36 weeks' gestation (HYPITAT): a multicentre, open-label randomised controlled trial. Lancet. 2009;374:979-988. doi: 10.1016/S0140-6736(09)60736-4.

29. Shields LE, Wiesner S, Klein C, Pelletreau B, Hedriana HL. Early standardized treatment of critical blood pressure elevations is associated with a reduction in eclampsia and severe maternal morbidity. Am J Obstet Gynecol. 2017;216:415.e1-415.e5. doi: 10.1016/j.ajog.2017.01.008. 